\title{
ELUSIVE ETHYLENE DETECTED IN SATURN'S NORTHERN STORM REGION
}

\author{
B. E. Hesman ${ }^{1,9}$, G. L. BjorakeR ${ }^{2}$, P. V. Sada ${ }^{3,10}$, R. K. Achterberg ${ }^{1,9}$, D. E. Jennings ${ }^{4,10}$, P. N. Romani ${ }^{2}$, \\ A. W. Lunsford ${ }^{5,10}$, L. N. FletcheR ${ }^{6}$, R. J. Boyle ${ }^{7} 10$, A. A. Simon-Miller ${ }^{8}$, C. A. Nixon ${ }^{1,9}$, AND P. G. J. Irwin ${ }^{6}$ \\ ${ }^{1}$ Department of Astronomy, University of Maryland, College Park, MD 20742, USA; brigette.e.hesman@nasa.gov \\ ${ }^{2}$ NASA/GSFC Code 693, Greenbelt, MD 20771, USA \\ ${ }^{3}$ Departamento de Física y Matemáticas, Universidad de Monterrey, Garza García, NL 66238, Mexico \\ ${ }^{4}$ NASA/GSFC Code 693 and Code 500, Greenbelt, MD 20771, USA \\ ${ }^{5}$ Department of Physics, Catholic University of America, Washington, DC 20064, USA \\ ${ }^{6}$ Atmospheric, Oceanic and Planetary Physics, Clarendon Laboratory, Parks Road, Oxford, OX1 3PU, UK \\ ${ }^{7}$ Department of Physics and Astronomy, Dickinson College, Carlisle, PA 17013, USA \\ ${ }^{8}$ NASA/GSFC Code 690, Greenbelt, MD 20771, USA \\ Received 2012 May 7; accepted 2012 September 28; published 2012 ???
}

\begin{abstract}
The massive eruption at $40^{\circ} \mathrm{N}$ (planetographic latitude) on Saturn in 2010 December has produced significant and lasting effects in the northern hemisphere on temperature and species abundances. The northern storm region was observed on many occasions in 2011 by Cassini's Composite Infrared Spectrometer (CIRS). In 2011 May, temperatures in the stratosphere greater than $200 \mathrm{~K}$ were derived from CIRS spectra in the regions referred to as "beacons" (warm regions in the stratosphere). Ethylene has been detected in the beacon region in Saturn's northern storm region using CIRS. Ground-based observations using the high-resolution spectrometer Celeste on the McMath-Pierce Telescope on 2011 May 15 were used to confirm the detection and improve the altitude resolution in the retrieved profile. The derived ethylene profile from the CIRS data gives a $\mathrm{C}_{2} \mathrm{H}_{4}$ mole fraction of $5.9 \pm 4.5 \times 10^{-7}$ at $0.5 \mathrm{mbar}$, and from Celeste data it gives $2.7 \pm 0.45 \times 10^{-6}$ at $0.1 \mathrm{mbar}$. This is two orders of magnitude higher than the amount measured in the ultraviolet at other latitudes prior to the storm. It is also much higher than predicted by photochemical models, indicating that perhaps another production mechanism is required or a loss mechanism is being inhibited.
\end{abstract}

Key words: planets and satellites: atmospheres - planets and satellites: individual (Saturn)

\section{INTRODUCTION}

Storms on Saturn probe the deep atmosphere as material is transported from levels beyond the reach of sunlight up to the observable atmosphere. Storms have a dramatic effect on the neighborhood, introducing sudden changes that dwarf the effects of seasonal change as Saturn progresses through its 29 year revolution about the Sun. In 2010 December, Saturn's northern hemisphere was spectacularly disrupted from its slow springtime warming by a massive storm eruption at approximately $40^{\circ} \mathrm{N}$ (planetographic latitude; Fletcher et al. 2011). This storm is only the sixth of its magnitude to ever be observed and the first one at this latitude in over a century. Saturn is known to have massive storm systems that erupt approximately once per saturnian year (29.4 Earth years), the most recent of which happened in 1990 September at $12^{\circ} \mathrm{N}$ (Sanchez-Lavega et al. 1991; Beebe et al. 1992). It is not known what triggers these large storms. The approximate annual periodicity suggests solar forcing, yet sunlight does not reach the water cloud where these storms are believed to originate (Dyudina et al. 2010; Hueso \& Sánchez-Lavega 2004). Saturn emits more radiation than it receives from the Sun, but the details of this process are not well understood. Storms may be a way in which Saturn releases its excess thermal energy in sudden bursts, rather than gradually. The convective plume that erupted in 2010 December was nearly 10 years earlier than expected in the cycle of great eruptions on Saturn (SanchezLavega et al. 1991). This convective plume was sheared to the north and south and its effects continued to disrupt the northern

9 Also at CRESST and NASA/GSFC Code 693, Greenbelt, MD 20771, USA.

${ }^{10}$ Also at National Solar Observatory. hemisphere throughout 2011. Due to technology limitations, studies of these massive storm systems in the thermal infrared have previously been impossible. Optical studies of these storms provide important information on morphology and cloud top locations. However, thermal infrared studies are essential to measuring the temperature and gas composition of the environment inside and adjacent to storm systems on Saturn.

Fortunately, the effects of the massive storm continued through at least 2011 July and the Composite Infrared Spectrometer (CIRS) on Cassini has produced an extensive record of the disturbance. This has been supplemented by groundbased observations at higher spectral resolution. Here we report the detection of ethylene in 2011 May using both CIRS and the ground-based spectrometer Celeste on the McMath-Pierce Telescope. Ethylene was detected in the warm beacon regions in Saturn's stratosphere. These are localized regions in latitude and longitude that are heated by $50 \mathrm{~K}$ or more compared with prestorm conditions. These stratospheric features are thought to be generated as a response to the upwelling of the storm (Fletcher et al. 2011). Previous infrared studies of Saturn's ethylene emission have shown it to be difficult to detect on Saturn (Encrenaz et al. 1975; Bézard et al. 2001), but the high temperatures in the beacon region have produced strong thermal emission at $10.5 \mu \mathrm{m}\left(950 \mathrm{~cm}^{-1}\right)$ due to ethylene in the stratosphere. A recent analysis of stellar occultation data acquired by the U1traviolet Imaging Spectrograph (UVIS) on Cassini has yielded a detection and vertical profile of ethylene between $0.1 \mu$ bar and 0.5 mbar in Saturn's atmosphere at $15.2 \mathrm{~N}$ (Shemansky \& Liu 2012). Comparisons of ethylene abundances derived in the storm region with other locations on Saturn provide insight into photochemical and dynamical processes occurring on this giant planet. 

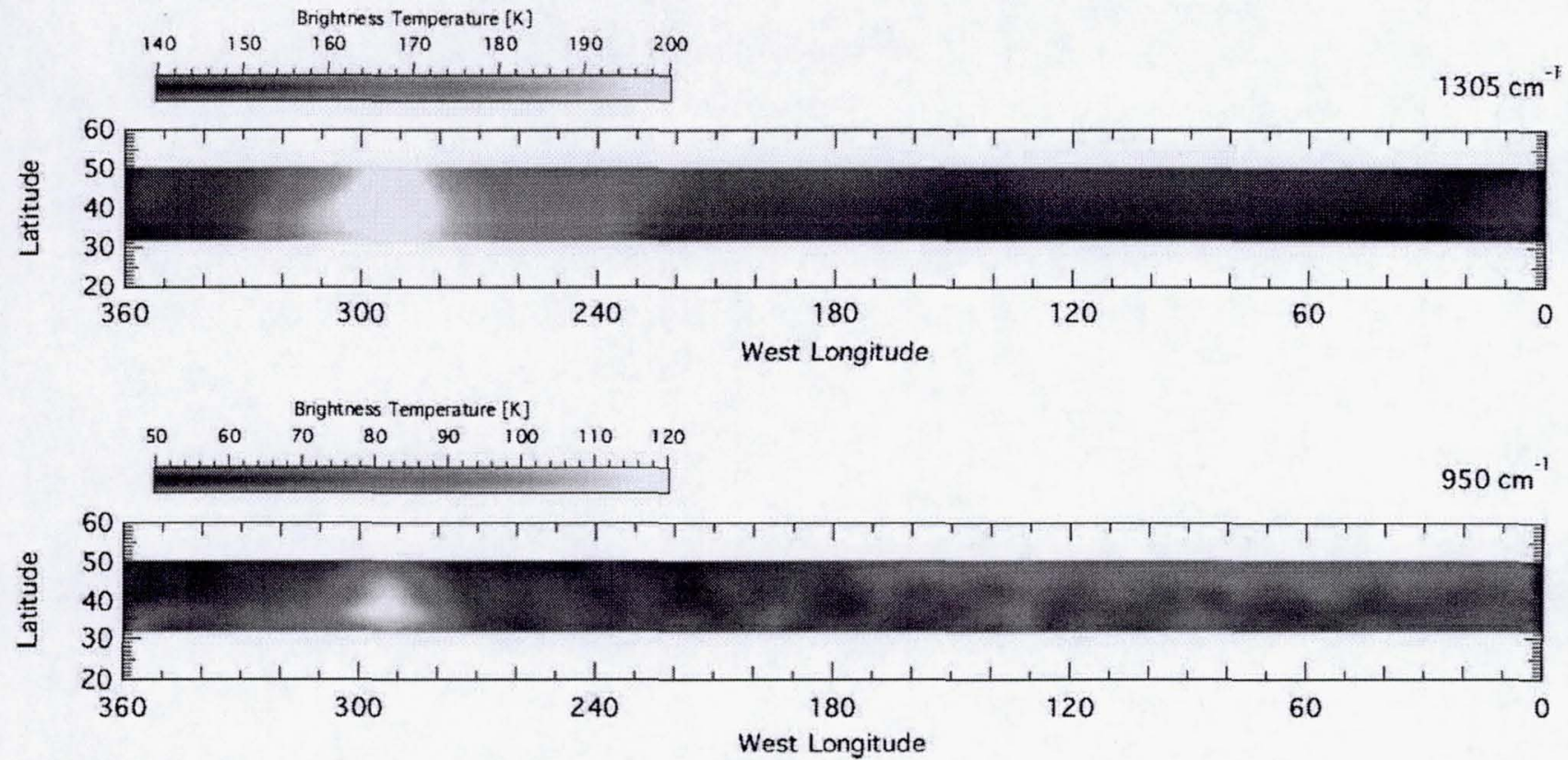

Figure 1. $2.5 \mathrm{~cm}^{-1}$ CIRS map over the northern storm latitudes from 2011 May 4. Top: this map shows data by plotting all of the FP4 pixels in the methane band at $1305 \mathrm{~cm}^{-1}$. Bottom: this map shows data by plotting all of the FP3 pixels in the ethylene region at $950 \mathrm{~cm}^{-1}$. The centroid of the hot stratospheric beacon is at $\sim 290^{\circ} \mathrm{W}$ longitude. Both maps have spatial resolutions of $2^{\circ}$ by $2^{\circ}$.

\section{OBSERVATIONS}

The combination of Cassini CIRS data with ground-based spectroscopy provides a powerful set of capabilities for studying hydrocarbons on Saturn. CIRS provides broad-band, absolutely calibrated spectra at high spatial resolution and modest spectral resolution. It also provides, through measurements of $\mathrm{CH}_{4}$ and collision induced $\mathrm{H}_{2}$ opacity, temperature profiles for both the stratosphere and upper troposphere of Saturn. The use of a cryogenic grating spectrometer (Celeste) operating at resolving powers of up to 30,000 on ground-based telescopes permits the detection of multiple emission lines of ethylene and yields improved altitude resolution in the retrieved vertical profile.

CIRS is a dual Fourier transform spectrometer covering the thermal infrared with three focal planes: the far-infrared focal plane, FP1, which is a single thermocouple detector covering $10-600 \mathrm{~cm}^{-1}$, and two mid-infrared focal planes, FP3 and FP4, which are arrays of $10 \mathrm{HgCdTe}$ detectors covering $600-1100 \mathrm{~cm}^{-1}$ and $1100-1500 \mathrm{~cm}^{-1}$, respectively (Flasar et al. 2005). The far-infrared detector operates at the instrument temperature of $170 \mathrm{~K}$, and the mid-infrared detectors operate at $80 \mathrm{~K}$. The fields of view per detector are $3.9 \mathrm{mrad}$ for the far-infrared and $0.3 \mathrm{mrad}$ for the mid-infrared. The apodized spectral resolution is selectable from 0.5 to $15 \mathrm{~cm}^{-1}$.

The CIRS observations were performed on 2011 May 4 using a spectral resolution of $2.5 \mathrm{~cm}^{-1}$. The spacecraft was targeted at $45^{\circ} \mathrm{N}$ (planetographic latitude) for an $11 \mathrm{hr}$ integration while the planet rotated across the field of view. This was followed by a second pointing at $35^{\circ} \mathrm{N}$ for an additional planetary rotation. The result was a cylindrical map of Saturn spanning $31^{\circ} \mathrm{N}-49^{\circ} \mathrm{N}$ over all longitudes, as shown in Figure 1. Calibration reference spectra were recorded by observing deep space and an internal warm source (the shutter of the instrument). Figure 1 shows the radiance (expressed in brightness temperature) in the methane band $\left(1305 \mathrm{~cm}^{-1}\right)$ as well as in the center of the ethylene band $\left(950 \mathrm{~cm}^{-1}\right)$. The maps of Figure 1 show that the beacon centroid is located near $290^{\circ} \mathrm{W}$ longitude and the mid-infrared map covered almost the full extent, in the north-south direction, of the storm region. Figure 2(a) shows the CIRS ethylene spectra inside (red curve) and outside (blue curve) the beacon region. The beacon region spectrum was created by averaging a total of 304 spectra from $3^{\circ}$ in latitude (average planetographic latitude of $38^{\circ}$ ) and $20^{\circ}$ in longitude (average longitude of $290^{\circ} \mathrm{W}$ - system III). The non-beacon spectrum was created by averaging a total of 3504 spectra from $3^{\circ}$ in latitude and $190^{\circ}$ in longitude (average longitude of $91^{\circ} \mathrm{W}$-system III). Ethylene is not present above the noise level in the non-beacon spectrum but appears as a single feature at $950 \mathrm{~cm}^{-1}$ in the beacon spectrum with a signal-to-noise ratio $(\mathrm{S} / \mathrm{N})$ of 8 .

Our ground-based instrument, Celeste, is a cryogenic grating spectrometer with an array detector that can achieve resolving powers up to 30,000 . This instrument has been described in Jennings et al. (2009). The instrument setup was similar to that used in previous observations (Romani et al. 2008; Hesman et al. 2009). The Celeste observations were performed at the McMath-Pierce Telescope on 2011 May 15. The spectrometer was coupled to the telescope with foreoptics which provided an approximately $\mathrm{f} / 8$ beam to the spectrometer entrance slit. The spectrometer uses an $18 \times 34 \mathrm{~cm}^{2}$ echelle grating and an Si:As detector array with a $128 \times 128$ pixel format and a spatial resolution of $\sim 1^{\prime \prime}$ pixel $^{-1}$. The entire spectrometer is cooled with liquid helium to an operating temperature of $6 \mathrm{~K}$.

For the Celeste observations the slit was oriented east-west on the planet and centered on $35^{\circ} \mathrm{N}$ latitude with a $300 \mu \mathrm{m}$ slit width to capture the spatial extent of the warm storm region. This translates into a slit length of $103^{\prime \prime}$ and a slit width of $4^{\prime \prime}$. The long slit was oriented in the east-west direction. Saturn was $\sim 19^{\prime \prime}$ during these observations, which is significantly smaller than the slit length of Celeste. Each resolution element subtended $33^{\circ}$ in latitude (slit width) and $10^{\circ}$ in longitude (along the slit). In addition, each time step covered $180^{\circ}$ in longitude and over a $3.5 \mathrm{hr}$ time period $300^{\circ}$ of longitude were sampled. This technique was used to guarantee continuous coverage 

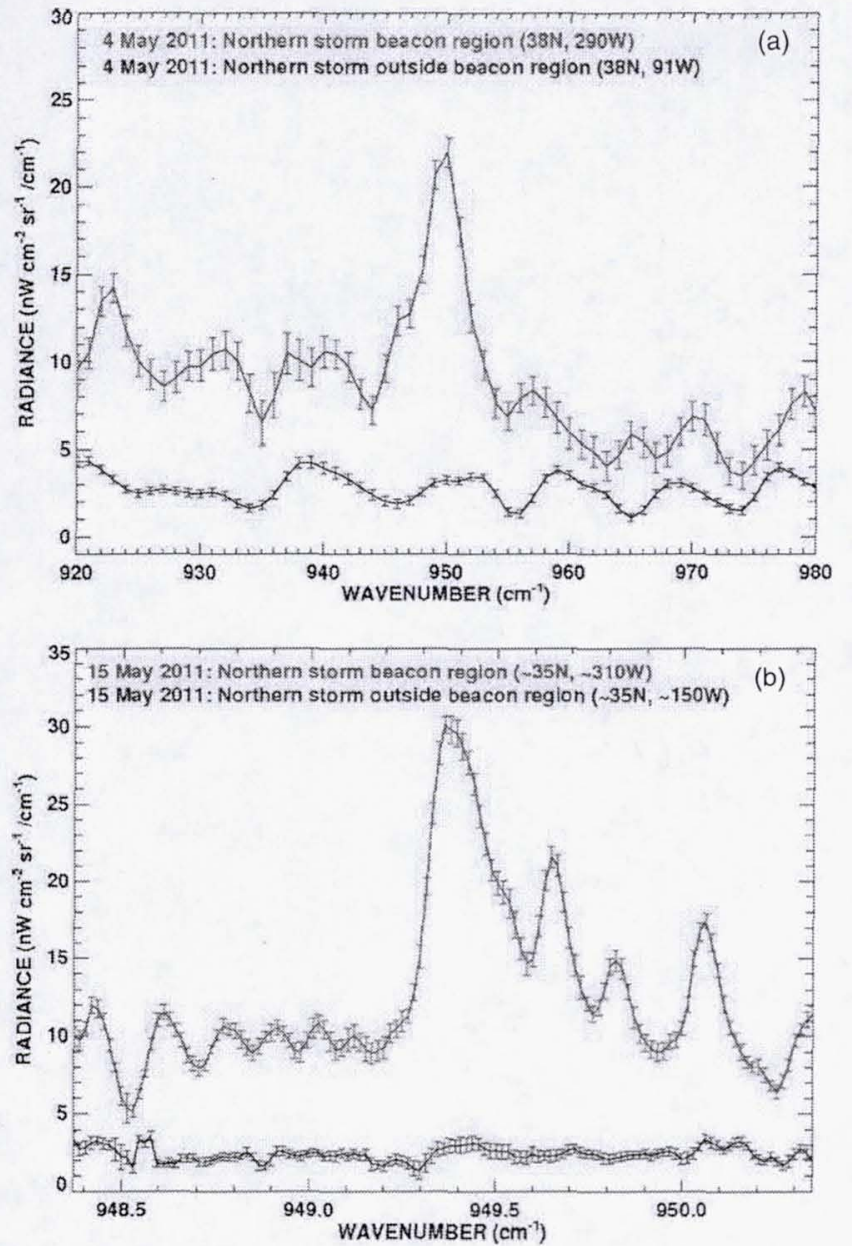

Figure 2. (a) The $2.5 \mathrm{~cm}^{-1}$ CIRS spectra at $38^{\circ} \mathrm{N}$ from 2011 May 4 inside the hot stratospheric beacon (red) and outside the region (blue). The CIRS spectra are shown over a $60 \mathrm{~cm}^{-1}$ interval. At this resolution, ethylene is detected as a single feature. (b) The $0.1 \mathrm{~cm}^{-1}$ Celeste spectra at $35^{\circ} \mathrm{N}$ from 2011 May 15 (McMath-Pierce Telescope) inside the hot stratospheric beacon (red) and outside the region (blue). The Celeste spectra are shown over a $2 \mathrm{~cm}^{-1}$ interval. Ethylene is detected as a band of lines at this resolution.

of the beacon as it moved across the dayside hemisphere of Saturn. This slit width gave a spectral resolution of $0.1 \mathrm{~cm}^{-1}$. The detector array covered a $2 \mathrm{~cm}^{-1}$ interval, centered on $949.4 \mathrm{~cm}^{-1}$. Sky subtraction was performed by nodding the telescope and the moon was used as a flat-field reference for calibration.

The Celeste observations were reduced in the standard method whereby the spectra from the two nod positions were subtracted to remove the offset signal, the response of the array was normalized by using the moon flat-fields, and the main $\mathrm{C}_{2} \mathrm{H}_{4}$ emission feature was shifted spatially between subtracted pairs (to account for rotation of the planet) and shifted spectrally (to account for the change in radial velocity as the planet rotated). A total of 12 subtracted pairs were averaged to create the $\mathrm{C}_{2} \mathrm{H}_{4}$ emission spectrum shown in Figure 2(b) (red curve). This spectrum represents a total of $3.4 \mathrm{hr}$ of integration time on the beacon region. In 2011 May, CIRS data from two successive maps indicate that the beacon was moving at $2^{\circ}$ day $^{-1}$. The beacon was therefore centered near $310^{\circ} \mathrm{W}$ longitude on May 15 during the Celeste observations. Ethylene is detected as a band of lines in this spectrum with an S/N of 17. This same method was applied in order to create the spectrum outside the beacon region using data from May 13th when the beacon was on the opposite side of the planet (blue curve). This spectrum represents a total of $4.5 \mathrm{hr}$ of integration time on the non-beacon side of Saturn. A scale factor for performing radiance calibration of the Celeste data was derived from modeling the continuum outside of the beacon region. As no ethylene was visible in the non-beacon data the continuum was modeled using known temperatures to derive a scale factor. The resulting scale factor was then applied to the beacon region. The uncertainty in the scale factor is estimated at $15 \%$.

\section{DATA ANALYSIS AND RESULTS}

Ethylene emission in the thermal infrared is coupled to both atmospheric temperature and the species abundance. The observed spectrum near $950 \mathrm{~cm}^{-1}$ consists of ethylene emission from the stratosphere riding on an optically thick continuum of collision-induced hydrogen produced in the troposphere. Thus, it is important to derive both the temperature in the troposphere and in the stratosphere from CIRS data. This temperature profile is then applied to models to retrieve the ethylene abundance profile in analyzing both the CIRS and Celeste spectra.

The temperature profile was retrieved using spectral regions separate from the ethylene region, in both the FP3 and FP4 focal planes. The same averaging used to produce the ethylene spectrum in the beacon region (as described in Section 2) was used to create the spectra used for temperature retrievals. Separate retrievals for upper tropospheric and stratospheric temperatures are performed using the constrained linear inversion algorithm described by Conrath et al. (1998). A detailed summary of the methods used in performing the temperature inversion can be found in Achterberg et al. (2008). For the tropospheric retrievals, the spectral ranges 600-620 and 640-660 $\mathrm{cm}^{-1}$ (FP3) are used where the atmospheric opacity is from the collision-induced S(1) line of hydrogen, assuming equilibrium hydrogen. Opacity from $\mathrm{H}_{2}-\mathrm{H}_{2}, \mathrm{H}_{2}-\mathrm{He}, \mathrm{H}_{2}-\mathrm{CH}_{4}$ pairs is included, using algorithms from Borysow et al. $(1985,1988)$ and Borysow \& Frommhold (1986). An $\mathrm{He} / \mathrm{H}_{2}$ ratio of 0.135 is assumed (Conrath \& Gautier 2000) along with a pressure dependent $\mathrm{CH}_{4}$ mole fraction profile based on the photochemical profile in Moses et al. (2000) scaled to a tropospheric value of $4.5 \times 10^{-3}$ as given in Flasar et al. (2005). The stratospheric temperatures are retrieved using the $v_{4}$ band of $\mathrm{CH}_{4}$ between 1250 and $1311 \mathrm{~cm}^{-1}$ (FP4). Methane transmittances were calculated using the correlated- $k$ method (Lacis \& Oinas 1991), using line data from the GEISA 2003 line atlas (Jacquinet-Husson et al. 2005) with $\mathrm{H}_{2} / \mathrm{He}$ broadening (Linda Brown, private communication). Figure 3(a) shows the temperature profile (solid black curve) retrieved from the beacon region in Saturn's northern storm region. The uncertainty limits in the retrieved temperature profile are approximately $1 \mathrm{~K}$ over the $0.5-5 \mathrm{mbar}$ range in the stratosphere and over the 100-300 mbar range in the troposphere. Between these pressure regions, the inversion algorithm smoothly interpolates temperatures. The temperature profile is not well determined at pressures below 0.5 mbar. However, the sensitivity of the ethylene retrievals to different temperatures at pressures less than 0.5 mbar was extensively tested. There is limited sensitivity to this altitude range in the ethylene line, and retrieved ethylene profile does not significantly change depending upon the input temperature in this altitude range.

The retrieved temperature profile in the beacon region was used in a photochemical model in order to generate an a priori abundance profile of the hydrocarbons present in the stratosphere (including ethylene). These theoretical profiles are 

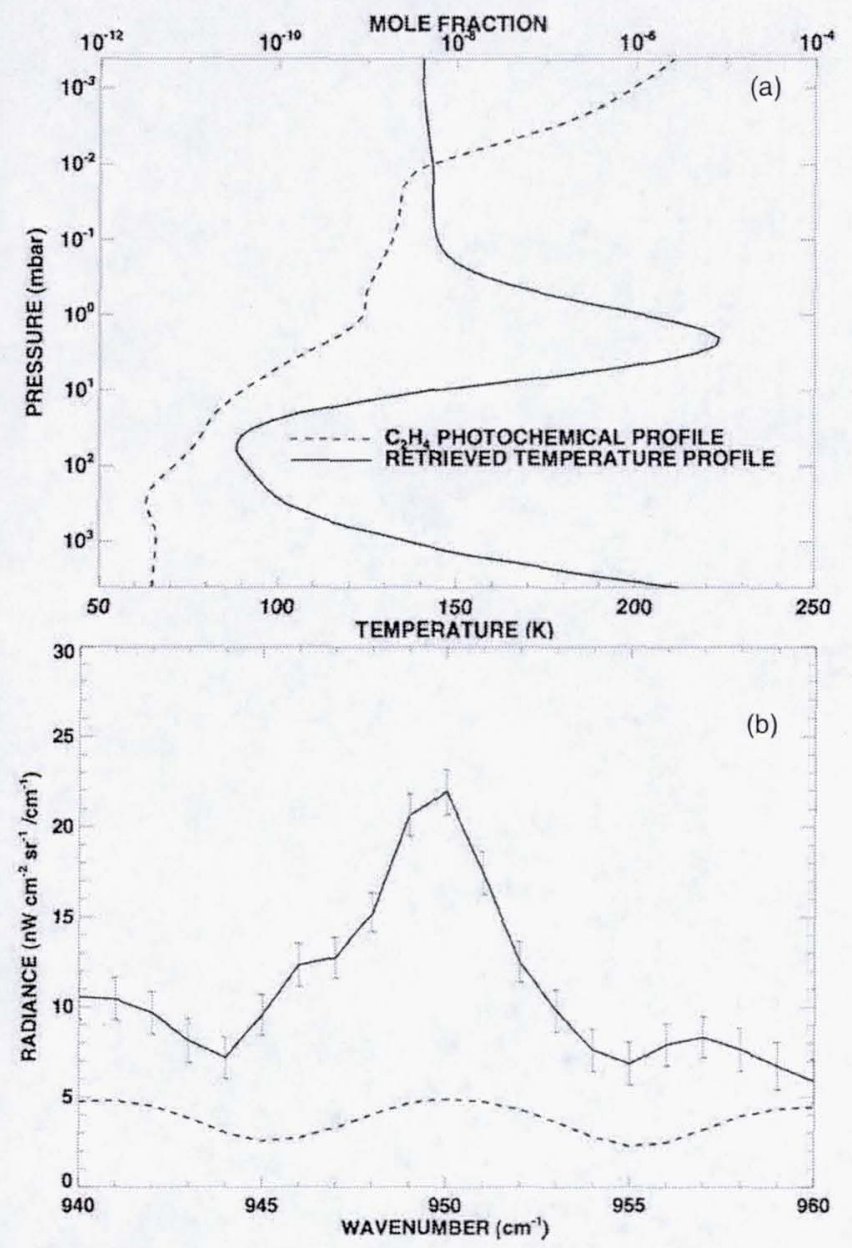

Figure 3. (a) The temperature profile as retrieved from the 2011 May CIRS data (black solid curve) and the calculated $\mathrm{C}_{2} \mathrm{H}_{4}$ photochemical profile produced using the temperature profile as input (black dashed curve). (b) The $2.5 \mathrm{~cm}^{-1}$ CIRS spectra at $38^{\circ} \mathrm{N}$ from 2011 May inside the hot stratospheric beacon (solid curve). The dashed curve is the model that results when the $\mathrm{C}_{2} \mathrm{H}_{4}$ photochemical profile and temperature profile are provided as input. Enhanced temperatures alone do not produce a measurable $\mathrm{C}_{2} \mathrm{H}_{4}$ line.

generated using a one-dimensional photochemical model. The model takes into account the photolysis and chemical reactions that interlink the hydrocarbons with each other and atomic hydrogen. It solves their coupled continuity equations assuming steady state conditions. The net flux of the species includes terms for both transport (eddy mixing) and molecular diffusion. For a more detailed model description of the model see Romani et al. (2008). Ethylene has a photochemical lifetime of about 20 days in the 2-0.2 mbar region. The photochemical model predicts $\mathrm{C}_{2} \mathrm{H}_{4}$ abundance profiles for the warmest $(220 \mathrm{~K})$ regions of Saturn's stratosphere that are enhanced by a factor of two in the 2-0.2 mbar altitude range over the ethylene abundances calculated for the unperturbed $(140 \mathrm{~K})$ atmosphere.

The $\mathrm{C}_{2} \mathrm{H}_{4}$ abundance profile retrievals were performed using the Non-Linear Optimal Estimator for Multivariate Spectral Analysis (NEMESIS) code as described in Irwin et al. (2008). Absorption of the contributing species was calculated using the correlated- $k$ method (Lacis \& Oinas 1991). The k-tables for $\mathrm{C}_{2} \mathrm{H}_{4}$ were calculated using line parameters based on data from the GEISA 2003 line atlas (Jacquinet-Husson et al. 2005) with modifications to the temperature exponent (set to 0.73 ) modified to use $\mathrm{H}_{2}$ pressure broadening rather than $\mathrm{N}_{2}$ (B. Bézard, private
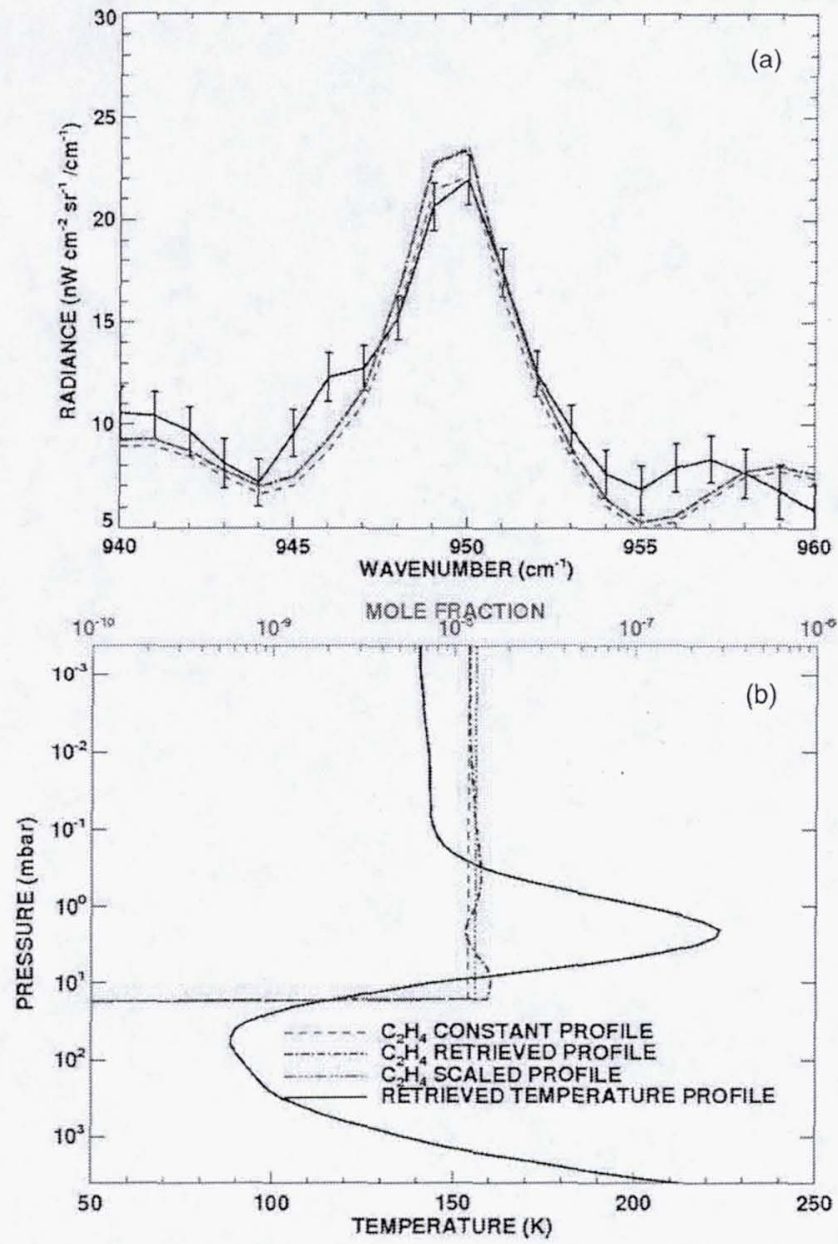

Figure 4. (a) The CIRS spectrum of ethylene (black solid curve) inside the hot stratospheric beacon. The colored curves are models based on $\mathrm{C}_{2} \mathrm{H}_{4}$ profiles with: a constant abundance of $12 \mathrm{ppb}$ (green); a retrieved profile starting from the constant abundance profile (blue); and, a scale factor retrieval starting from the constant abundance profile (red). (b) The temperature profile used in producing the model spectra shown in (a) (black), the colored curves are the $\mathrm{C}_{2} \mathrm{H}_{4}$ abundance profiles that produce the model spectra shown in (a).

communication). Inputs into the model were the temperature profiles derived from the data inside and outside the beacon region and the photochemical profiles of $\mathrm{C}_{2} \mathrm{H}_{4}$ calculated based on the derived temperature profiles.

In a first test, a forward model was run using the temperature profile retrieved from the CIRS data (Figure 3 top; solid curve) and a photochemical model of ethylene that was calculated using the retrieved temperature profile (Figure 3 top; dashed curve). In (Figure 3 bottom) we compare the observed CIRS spectrum of the beacon region with a synthetic spectrum calculated using the heated temperature profile and $\mathrm{C}_{2} \mathrm{H}_{4}$ profile shown in the top figure. It is clear that enhanced temperatures alone do not produce sufficient ethylene emission to match the observations. Thus, increasing the abundance of this species is needed in order to fit the CIRS data (Figure 3 bottom; solid curve).

In order to assess the information content in the CIRS and Celeste ethylene spectra an a priori profile that is constant with altitude until 20 mbar was used. This technique was used to determine how sensitive the model retrievals are to the starting conditions. In Figure 4, an ethylene mole fraction of $1.2 \times 10^{-8}$ was assumed for the constant profile (green curves). This profile produces a good fit to the CIRS data. In 

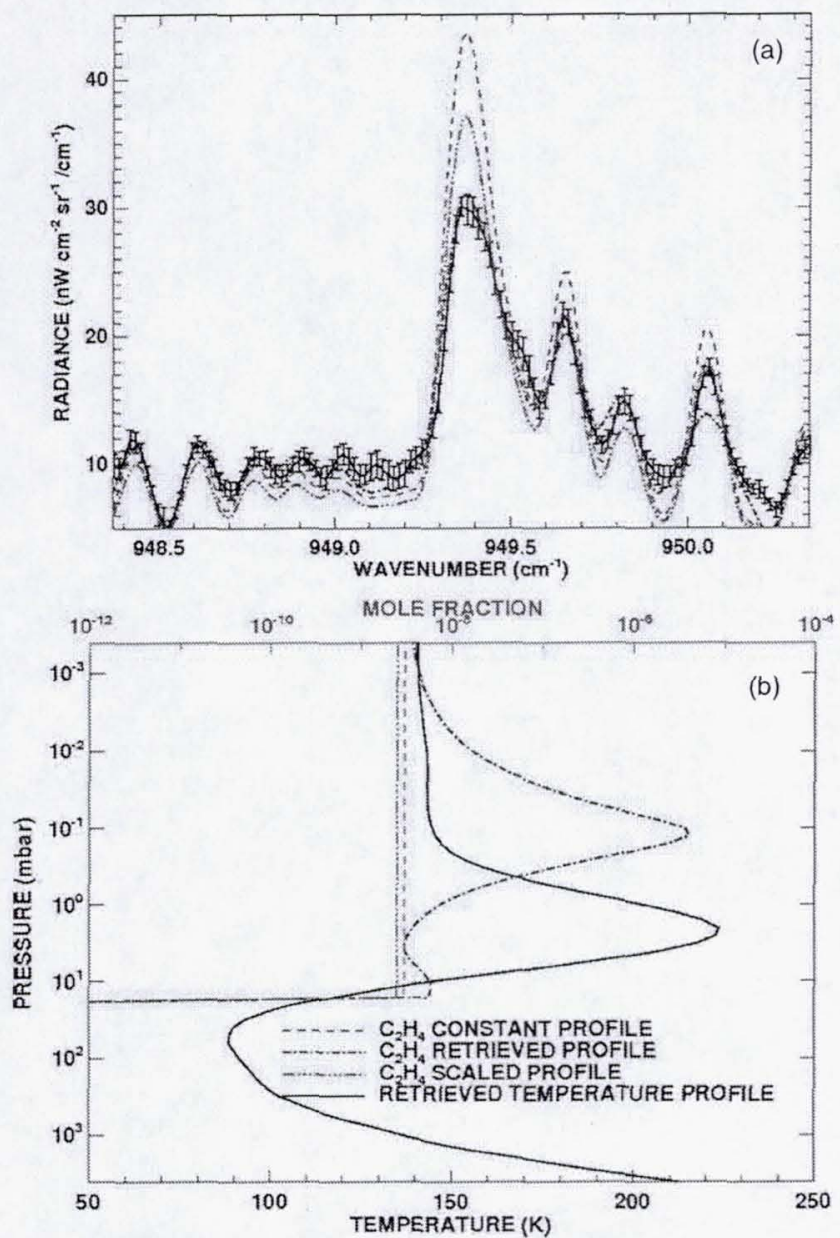

Figure 5. (a) The Celeste spectrum of ethylene (black solid curve) inside the hot stratospheric beacon. The colored curves are models based on $\mathrm{C}_{2} \mathrm{H}_{4}$ profiles with: a constant abundance of $3 \mathrm{ppb}$ (green); a retrieved profile starting from the constant abundance profile (blue); and, a scale factor retrieval starting from the constant abundance profile (red). (b) The temperature profile used in producing the model spectra shown in (a) (black), the colored curves are the $\mathrm{C}_{2} \mathrm{H}_{4}$ abundance profiles that produce the model spectra shown in (a).

the other two cases the constant profile was allowed to vary continuously (blue) and with a scale factor (red) in order to produce the best-fit to the CIRS line. The profiles that result are not significantly different from the constant abundance with height profile. In Figure 5, a constant with height profile with an abundance of $3 \times 10^{-9}$ is used as the a priori profile (green curves) to fit the Celeste data. This profile does not produce a spectrum that fits any of the ethylene lines in the Celeste spectrum (Figure 5(a)). A best-fit profile was retrieved using a scale factor adjustment (red curve) which fits the ethylene lines at $949.65 \mathrm{~cm}^{-1}$ and $950.05 \mathrm{~cm}^{-1}$, but does not fit the main ethylene line at $949.35 \mathrm{~cm}^{-1}$. When a best-fit profile was derived by allowing the a priori constant profile to vary continuously in altitude the blue curve in Figure 5(b) results. This profile produces the best-fit overall to the entire Celeste spectrum. These results have motivated continuous profiles to be retrieved from both data sets using the photochemical profile as an input.

In performing the retrievals from the CIRS data the temperature profiles were fixed but the calculated photochemical profiles were used as the initial prediction and allowed to vary in order to fit the emission in the CIRS spectra. NEMESIS was used to
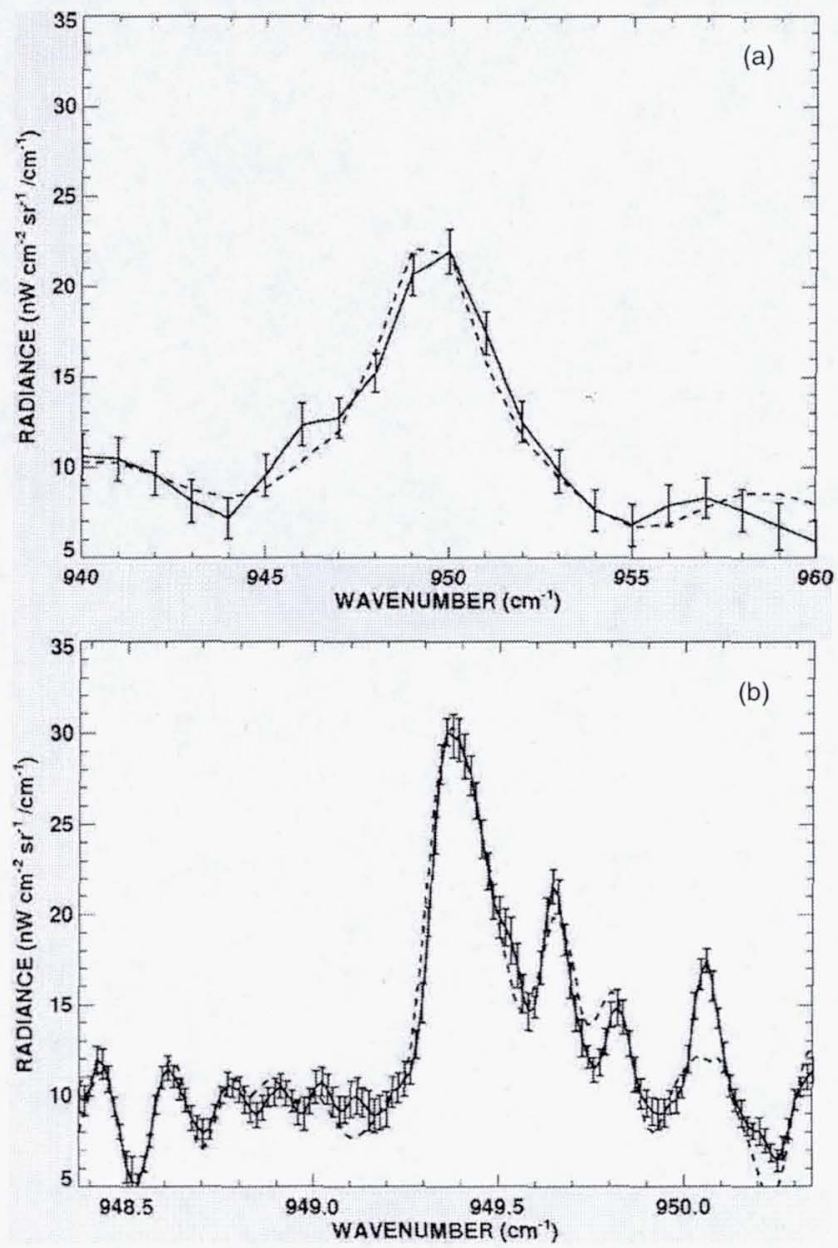

Figure 6. (a) The CIRS spectrum of ethylene (black solid curve) inside the hot stratospheric beacon. The dashed curve is the best-fit model to the data. (b) The Celeste spectrum of ethylene (black solid curve) inside the hot stratospheric beacon. The dashed curve is the best-fit model to the data.

adjust the photochemical profiles between 0.03 and 3 mbar in order to retrieve the ethylene abundance in this pressure range. Figure 6 shows the ethylene spectra from CIRS and Celeste along with their best-fit model spectra. In both cases the data are fit very well with the model. In Figure 6(b) there is an excellent fit of the model to the peak emission in the Celeste spectrum. However, the poor fit around the ethylene feature at $950.1 \mathrm{~cm}^{-1}$ may be the result of incomplete laboratory data for ethylene at this wavenumber.

Figure 7 (top) shows the retrieved ethylene profile from the CIRS data (solid green curve). The dashed green curve is the photochemical profile computed from the retrieved temperature profile shown in black. This profile was used as the a priori input into NEMESIS. The error bars are shown only for the pressures over which the retrieval is sensitive based on the altitude probed by the $\mathrm{C}_{2} \mathrm{H}_{4}$ line present in the CIRS data. The peak abundance found from the CIRS data is $5.9 \pm 4.5 \times 10^{-7}$ at 0.5 mbar. The uncertainties were calculated using NEMESIS which takes into account both the measured error in the spectrum and an appropriate amount of forward modeling error. This includes, for example, uncertainties based on the line parameters and in the correlated- $k$ model (see Irwin et al. 2008).

In performing the retrievals from the Celeste data the temperature profiles were also held fixed but in this case the $\mathrm{C}_{2} \mathrm{H}_{4}$ 


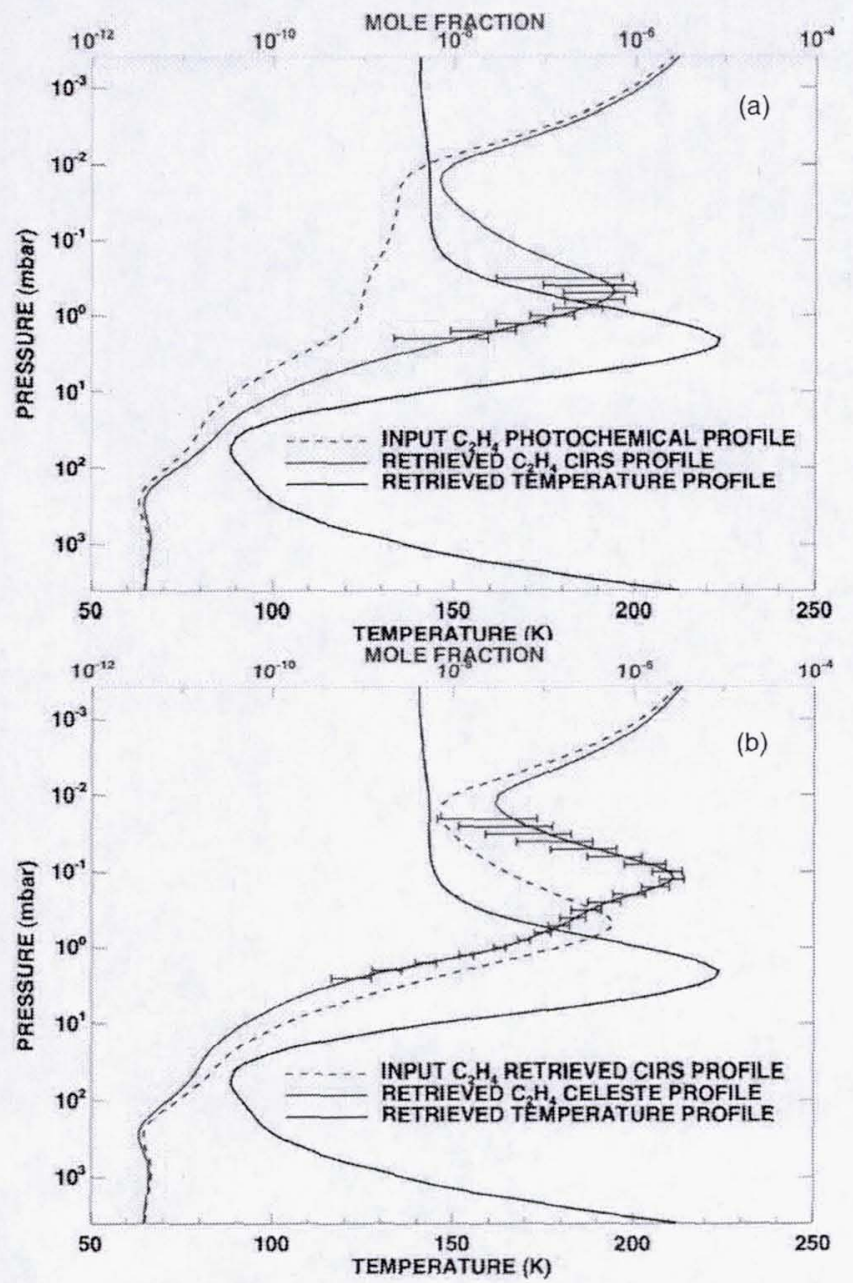

Figure 7. (a) Retrieval of ethylene (green solid curve) from $2.5 \mathrm{~cm}^{-1}$ CIRS spectra from 2011 May inside the hot stratospheric beacon. The dashed curve indicates the abundance profile of ethylene as determined by photochemistry alone. The black curve shows the temperature profile retrieved from these data. (b) Retrieval of ethylene (green solid curve) from $0.1 \mathrm{~cm}^{-1}$ Celeste data from 2011 May (McMath-Pierce Telescope) inside the hot stratospheric beacon. The retrieved ethylene profile from the CIRS data was used as the starting point in this case (green dashed curve). The higher spectral resolution of the Celeste data and the combination of it with the CIRS retrieved ethylene profile allow more altitude information to be retrieved about the ethylene profile. Where there is no information in altitude the profiles return to the input photochemical profiles. In both retrievals, the uncertainty limits indicate the altitude over which the retrieval is statistically reliable.

profile retrieved from the CIRS data was used as the a priori profile (Figure 7 top; solid green curve). NEMESIS was used to adjust the a priori profile between 0.03 and 3 mbar in order to retrieve the ethylene abundance in this pressure range. Figure 7 (bottom) shows the retrieved ethylene profile from the Celeste data (solid green curve). Ethylene still shows a greatly enhanced abundance over the photochemical profile with a peak abundance of $2.7 \pm 0.45 \times 10^{-6}$ at 0.1 mbar. The band of ethylene lines present in the Celeste data and the initial starting condition of the retrieved CIRS profile resulted in the abundance profile being retrieved over a broader range of altitudes. The uncertainties in the profile derived from the Celeste data were calculated using the same procedure as used in determining the uncertainties in the profile derived from the CIRS data.

\section{DISCUSSION}

The detection of ethylene in the northern storm region is significant for two reasons. First of all, this short-lived hydrocarbon is a tracer of chemistry occurring in an unusual, dynamical region of Saturn's stratosphere. In addition, prior ground-based searches for ethylene in the thermal infrared have resulted only in tentative detections on Saturn (Encrenaz et al. 1975; Bézard et al. 2001). Ethylene has never been detected by CIRS prior to the eruption of the northern storm. A significant finding from these measurements is that calculations based on photochemistry do not produce an ethylene profile that when combined with the increased beacon temperatures produces a measurable ethylene line. Emission from all of the hydrocarbons is expected to increase in the beacon regions because of the extreme temperatures found there (these results show approximately $220 \mathrm{~K}$ at 2 mbar). Abundance increases in ethylene of approximately two times the quiescent amount are expected from photochemical modeling. This, however, would have produced no measurable ethylene line in either the CIRS or Celeste data, as shown in Figure 3(b) for CIRS. In addition, the recent analysis of stellar occultation data acquired by UVIS yielded vertical profiles of ethylene at two different latitudes $\left(15^{\circ} .2 \mathrm{~N}\right.$ and $\left.42^{\circ} .7 \mathrm{~S}\right)$ in the upper atmosphere of Saturn (Shemansky \& Liu 2012). The UV measurements of ethylene show good agreement with photochemical models near the $1 \mu$ bar level where $\mathrm{C}_{2} \mathrm{H}_{4}$ is produced from photolysis of $\mathrm{CH}_{4}$. Only the profile at $15^{\circ} \mathrm{N}$ extended to deep enough levels (0.5 mbar) for comparison with CIRS data. Our results show $\mathrm{C}_{2} \mathrm{H}_{4}$ abundances in the storm at $40^{\circ} \mathrm{N}$ two orders-of-magnitude larger than that derived from UVIS observations at $15^{\circ} \mathrm{N}$ in 2006 , well before the northern storm.

The significant departure of the ethylene profile found in this study from the photochemical prediction required a thorough investigation. CIRS data are less sensitive to temperatures at pressures less than approximately $0.1 \mathrm{mbar}$. Therefore as an additional test, temperature profiles with significant deviations ( $\pm 20 \mathrm{~K}$ at pressures less than $0.1 \mathrm{mbar}$ ) were used as input when deriving the ethylene profile from the CIRS data. In all situations, it was found that there was less than a $10 \%$ difference between the derived ethylene profiles indicating that the $950 \mathrm{~cm}^{-1}$ region is not probing altitudes where the pressure is less than 0.1 mbar. In another test, synthetic spectra were calculated using a scaling factor to the ethylene photochemical profile. Here the shape of the photochemical profile is preserved, but the $\mathrm{C}_{2} \mathrm{H}_{4}$ mole fraction is enhanced at all altitudes. In this case, the overall fit produced was of poorer quality than the fit that allowed the profile to vary continuously. A scaling factor of 91 was needed in order to fit the observed ethylene emission using the photochemical profile.

The hot beacon regions in the stratosphere are a reaction to the forceful eruption of the storm into the upper troposphere. The tropospheric storm occurred at a latitude in which quasistationary planetary waves are able to propagate upward from the tropopause into the stratosphere (Achterberg \& Flasar 1996). Adiabatic heating in the downward phase of a wave would cause heating of the observed magnitude $(\sim 60 \mathrm{~K})$ with downward mass motion of approximately one-half of a pressure scale height, which is insufficient to cause the observed 100-fold increase in ethylene. Radiative cooling during the descent would allow transport from higher altitudes, but because the radiative timescale is roughly an order of magnitude larger than the photochemical timescale for ethylene (Conrath et al. 1990), vertical velocities consistent with the observed temperature 
perturbation would produce minimal deviation of the ethylene profile from photochemical equilibrium. Thus, the enhanced ethylene emission in the beacon cannot be explained simply by transport of ethylene from higher altitudes.

Ethylene is known to be primarily formed through two reaction schemes. The first being

$$
\begin{aligned}
& \mathrm{CH}_{4}+h v \rightarrow \mathrm{CH}+\mathrm{H}_{2}+\mathrm{H}, \\
& \mathrm{CH}+\mathrm{CH}_{4} \rightarrow \mathrm{C}_{2} \mathrm{H}_{4}+\mathrm{H},
\end{aligned}
$$

where $\mathrm{CH}$ is the intermediate in this two-step process. The second is a three-step process involving the ground-state (triplet) ${ }^{3} \mathrm{CH}_{2}$ :

$$
\begin{aligned}
& \mathrm{CH}_{4}+h v \rightarrow{ }^{3} \mathrm{CH}_{2}+2 \mathrm{H}, \\
& { }^{3} \mathrm{CH}_{2}+\mathrm{H} \rightarrow \mathrm{CH}+\mathrm{H}_{2}, \\
& \mathrm{CH}+\mathrm{CH}_{4} \rightarrow \mathrm{C}_{2} \mathrm{H}_{4}+\mathrm{H} .
\end{aligned}
$$

These processes indicate that the production rate of ethylene is directly proportional to $\mathrm{CH}_{4}$ photolysis rate at Ly $\alpha$ (Romani et al. 2008). Potentially, there could be a third mechanism producing ethylene:

$$
\mathrm{C}_{2} \mathrm{H}_{3}+\mathrm{H}_{2} \rightarrow \mathrm{C}_{2} \mathrm{H}_{4}+\mathrm{H}
$$

(Allen et al. 1992). Current laboratory measurements indicate that the energy for this reaction is too large to become significant by the increase in temperature observed in the beacon.

The altitude range where the increased ethylene is measured from these data sets is primarily a sink region via the following reaction:

$$
\mathrm{C}_{2} \mathrm{H}_{4}+\mathrm{H}+\mathrm{M} \rightarrow \mathrm{C}_{2} \mathrm{H}_{5}+\mathrm{M}
$$

(Romani et al. 2008). It may be possible that this reaction is being inhibited in the region where the significantly higher ethylene is observed. Because ethylene leads directly to the formation of other more prominent hydrocarbons, such as ethane, understanding how it is created and destroyed is important to understanding the photochemistry in Giant Planet atmospheres.

Additional CIRS and Celeste observations acquired later in 2011 and 2012 will permit us to observe the evolution of the emission from ethylene and other hydrocarbons in the year following the appearance of the first storm clouds. These observations are key to unlocking the mystery of why ethylene is greatly enhanced in the storm region compared with photochemical predictions.

The authors thank the Cassini/CIRS calibration team for their assistance in the calibration of these data sets. The authors also thank the McMath-Pierce Telescope staff for their assistance during these observations. This research was supported by the NASA Cassini/CIRS project, by the NASA Planetary Astronomy (PAST) Program grant number NNX11AJ47G, and the NASA Cassini Data Analysis Participating Scientists (CDAPS) Program grant number NNX12AC24G. The National Solar Observatory is operated by the Association of Universities for Research in Astronomy under contract for the National Science Foundation.

Facilities: McMath-Pierce, Cassini

\section{REFERENCES}

Achterberg, R. K., Conrath, B. J., Gierasch, P. J., Flasar, F. M., \& Nixon, C. A. 2008, lcarus, 194, 263

Achterberg, R. K., \& Flasar, F. M. 1996, lcarus, 119, 350

Allen, M., Yung, Y. L., \& Gladstone, G. R. 1992, lcarus, 100, 527

Beebe, R. F., Barnet, C., Sada, P. V., \& Murrell, A. S. 1992, Icarus, 95, 163

Bézard, B., Moses, J. I., Lacy, J., et al. 2001, BAAS, 33, 1079

Borysow, A., \& Frommhold, L. 1986, ApJ, 304, 849

Borysow, J., Frommhold, L., \& Birnbaum, G. 1988, ApJ, 326, 509

Borysow, J., Trafton, L., Frommhold, L., \& Birnbaum, G. 1985, Apl, 296, 644

Conrath, B. J., \& Gautier, D. 2000, learus, 144, 124

Conrath, B. J., Gierasch, P. J., \& Leroy, S. S. 1990, Icantus, 83, 255

Conrath, B. J., Gierasch, P. J., \& Ustinov, E. A. 1998, Icartis, 135, 501

Dyudina, U. A., Ingersoll, A. P., Ewald, S. P., et al. 2010, Geophys. Res. Lett, 37, L09205

Encrenaz, T., Combes, M., Zeau, Y., Vapillon, L., \& Berezne, J. 1975, A\&A, 42,355

Flasar, F. M., Achterberg, R. K., Conrath, B. J., et al. 2005, Science, 307, 1247

Fletcher, L. N., Hesman, B. E., Irwin, P. G. J., et al. 2011, Sclence, 332, 1413

Hesman, B. E., Jennings, D. E., Sada, P. V., et al. 2009, lcurus, 202. 249

Hueso, R., \& Sánchez-Lavega, A. 2004, Icarus, 172, 255

Irwin, P. G. J., Teanby, N. A., de Kok, R., et al. 2008, J. Quant. Spectrosc. Radiat. Transfer, 109, 1136

Jacquinet-Husson, N., Scott, N. A., Chédin, A., et al. 2005, J. Quant. Spectrose: Radiat. Transfer, 95, 429

Jennings, D. E., Romani, P. N., Bjoraker, G. L., et al. 2009, J. Phys. Chem. A, 113,11101

Lacis, A. A., \& Oinas, V. 1991, J. Geopliys. Res., 96. 9027

Moses, J. I., Bézard, B., Lellouch, E., et al. 2000, Icarus, 143, 244

Romani, P. N., Jennings, D. E., Bjoraker, G. L., et al. 2008, Icarus, 198, 420

Sanchez-Lavega, A., Colas, F., Lecacheux, J., et al. 1991, Nature, 353, 397

Shemansky, D., \& Liu, X. 2012, Can. J. Phys., 90, 817 


\section{Queries}

\section{Page 1}

Q1

Author: The "expansion" of affiliation footnote "10" has been moved to the acknowledgments. Please check for the correctness.

\section{Page 4}

Q2

Author: Please provide year in reference citation "B. Bézard, private communication."

\section{Page 6}

Q3

Author: Please confirm if the edit made to the sentence "A significant finding from these..." retains the intended sense.

\section{Page 7}

Q4

Author: Please check the details for any journal references that do not have a pale purple link (CrossRef doi) or a blue link (NASA ADS or arXiv e-print) in the two-column proof (articlestyle layout). A journal reference with no links may contain some incorrect information.

\section{Online-only colour figures}

This proof PDF is identical in specification to the PDF file that will be published in the online journal. To view any online-only color figures as they will appear in the printed journal, we recommend that this color PDF file be printed on a black \& white printer. 